\title{
Computing volume of solids bounded by Bézier surfaces
}

\author{
Imre Juhász
}




\title{
COMPUTING VOLUME OF SOLIDS BOUNDED BY BÉZIER SURFACES
}

\author{
IMRE JUHÁSZ \\ Department of Descriptive Geometry, University of Miskolc \\ 3515 Miskolc - Egyetemváros, Hungary \\ agtji@gold.uni-miskolc.hu
}

[Received June 22, 2000]

\begin{abstract}
We provide an optimized closed formula for the volume of the solid, bounded by a Bézier surface and the cones determined by its boundary curves and the origin. The obtained formula is easy to implement in programming languages, since it contains only basic arithmetic operations.
\end{abstract}

Mathematical Subject Classification: 51M25, 68U07

Keywords: Bézier surface, volume

\section{Introduction}

Bézier curves and surfaces play a significant role in Computer Aided Geometric Design (CAGD), therefore their specification, modification and different properties are of interest. Specification and modification of Bézier objects are well explored fields of CAGD but there are some special problems to be solved, such as to find the optimal way of computing the volume of solids bounded by Bézier surfaces. Following an elementary approach, we derive an exact closed formula which can substitute approximating numerical procedures. This task can be considered as a spatial generalization of the problem of computing the signed area of a plane figure bounded by Bézier curves, cf. [3].

In this paper we consider Bézier surfaces of the following definition:

Definition 1 The tensor product surface defined by the equation

$$
\mathbf{b}(u, v)=\sum_{i=0}^{n} \sum_{j=0}^{m} \mathbf{b}_{i j} B_{i}^{n}(u) B_{j}^{m}(v), u \in[0,1], v \in[0,1]
$$

is called Bézier surface, where $\mathbf{b}_{i j} \in \mathbb{R}^{3}$ are the control points of the surface and $B_{i}^{n}(u)=\left(\begin{array}{c}n \\ i\end{array}\right) u^{i}(1-u)^{n-i},(i=0, \ldots, n)$ is the $i^{\text {th }}$ Bernstein polynomial of degree $n$. 


\section{Volume calculation}

Theorem 2 The signed volume of the solid bounded by the Bézier surface (1.1) and the cones determined by its boundary curves and the origin is

$$
V=\frac{1}{3(3 n-1)(3 m-1)} \sum_{r=0}^{z-3} \sum_{s=r+1}^{z-2} \sum_{t=s+1}^{z-1} \beta_{i j k l p q}^{m n}\left(\mathbf{b}_{i j}, \mathbf{b}_{k l}, \mathbf{b}_{p q}\right)
$$

where $\left(\mathbf{b}_{i j}, \mathbf{b}_{k l}, \mathbf{b}_{p q}\right)$ denotes the mixed product of the specified vectors, $z=(n+1)(m+1)$,

$$
\begin{aligned}
j & =r \bmod (m+1), i=(r-j) /(m+1), \\
l & =s \bmod (m+1), k=(s-l) /(m+1), \\
q & =t \bmod (m+1), p=(t-q) /(m+1),
\end{aligned}
$$

and

$$
\begin{aligned}
\beta_{i j k l p q}^{m n}= & \frac{l(i-p)+q(k-i)+j(p-k)}{\left(\begin{array}{c}
3 n-2 \\
i+k+p-1
\end{array}\right)\left(\begin{array}{c}
3 m-2 \\
j+l+q-1
\end{array}\right)} \\
& \left(\begin{array}{l}
n \\
i
\end{array}\right)\left(\begin{array}{l}
n \\
k
\end{array}\right)\left(\begin{array}{l}
n \\
p
\end{array}\right)\left(\begin{array}{l}
m \\
j
\end{array}\right)\left(\begin{array}{l}
m \\
l
\end{array}\right)\left(\begin{array}{l}
m \\
q
\end{array}\right) .
\end{aligned}
$$

Proof. First we determine the signed volume $V$ of the solid bounded by the surface $\mathbf{s}(u, v), u \in\left[u_{0}, u_{1}\right], v \in\left[v_{0}, v_{1}\right]$ and the cones determined by its boundary curves and the origin. For this purpose we divide the interval $\left[u_{0}, u_{1}\right]$ into $c$, and $\left[v_{0}, v_{1}\right]$ into $d$ equal parts. The corresponding parameter lines divide the surface into rectangular patches. We approximate the volume of the solid determined by such a patch and the origin with tetrahedra of Figure 1. Their volumes are

$$
\begin{aligned}
V_{i j} & =\frac{1}{6}\left(\mathbf{s}\left(u_{i+1}, v_{j}\right)-\mathbf{s}\left(u_{i}, v_{j}\right), \mathbf{s}\left(u_{i}, v_{j+1}\right)-\mathbf{s}\left(u_{i}, v_{j}\right), \mathbf{s}\left(u_{i}, v_{j}\right)\right) \text { and } \\
V^{i j} & =\frac{1}{6}\left(\mathbf{s}\left(u_{i+1}, v_{j+1}\right)-\mathbf{s}\left(u_{i+1}, v_{j}\right), \mathbf{s}\left(u_{i+1}, v_{j+1}\right)-\mathbf{s}\left(u_{i}, v_{j+1}\right), \mathbf{s}\left(u_{i+1}, v_{j+1}\right)\right) .
\end{aligned}
$$

Denoting the sum of the appropriate tetrahedra by $V_{c d}$ and $V^{c d}$ we obtain

$$
V_{c d}=\sum_{i=0}^{c-1} \sum_{j=0}^{d-1} V_{i j}, V^{c d}=\sum_{i=0}^{c-1} \sum_{j=0}^{d-1} V^{i j} \text { and } \lim _{\substack{c \rightarrow \infty \\ d \rightarrow \infty}}\left(V_{c d}+V^{c d}\right)=V,
$$

moreover, the equalities

$$
\lim _{\substack{c \rightarrow \infty \\ d \rightarrow \infty}} V_{c d}=\lim _{\substack{c \rightarrow \infty \\ d \rightarrow \infty}} V^{c d}=\frac{1}{6} \int_{u_{0}}^{u_{1}} \int_{v_{0}}^{v_{1}}\left(\frac{\partial}{\partial u} \mathbf{s}(u, v), \frac{\partial}{\partial v} \mathbf{s}(u, v), \mathbf{s}(u, v)\right) d u d v
$$


hold, thus

$$
V=\frac{1}{3} \int_{u_{0}}^{u_{1}} \int_{v_{0}}^{v_{1}}\left(\frac{\partial}{\partial u} \mathbf{s}(u, v), \frac{\partial}{\partial v} \mathbf{s}(u, v), \mathbf{s}(u, v)\right) d u d v
$$

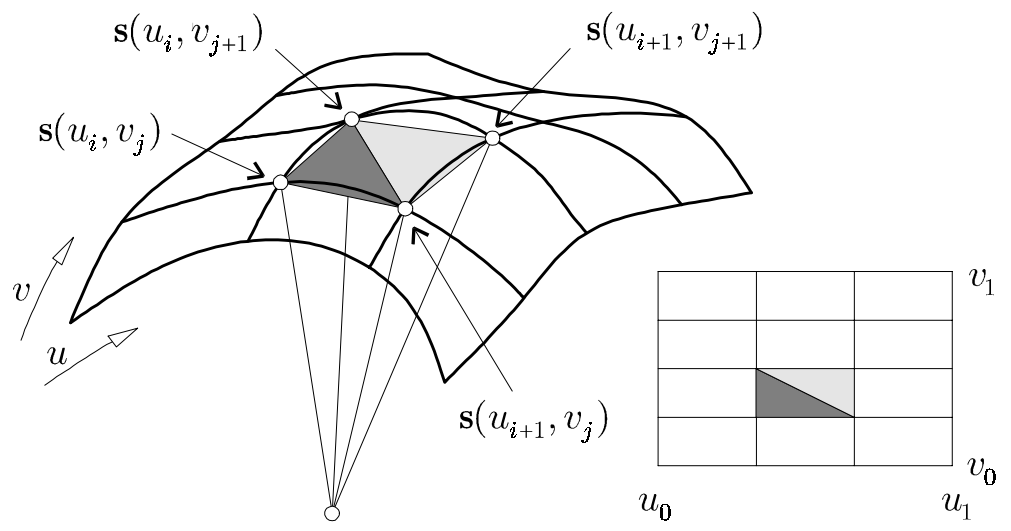

Figure 1. The approximating tetrahedra

The partial derivatives of the Bézier surface of Equation (1.1) are

$$
\begin{aligned}
\frac{\partial}{\partial u} \mathbf{b}(u, v) & =n \sum_{i=0}^{n} \sum_{j=0}^{m} \mathbf{b}_{i j}\left(B_{i-1}^{n-1}(u)-B_{i}^{n-1}(u)\right) B_{j}^{m}(v), \\
\frac{\partial}{\partial v} \mathbf{b}(u, v) & =m \sum_{k=0}^{n} \sum_{l=0}^{m} \mathbf{b}_{k l} B_{k}^{n}(u)\left(B_{l-1}^{m-1}(v)-B_{l}^{m-1}(v)\right)
\end{aligned}
$$

and the required volume is

$$
\begin{gathered}
V=\frac{n m}{3} \int_{0}^{1} \int_{0}^{1}\left(\sum_{i=0}^{n} \sum_{j=0}^{m} \mathbf{b}_{i j}\left(B_{i-1}^{n-1}(u)-B_{i}^{n-1}(u)\right) B_{j}^{m}(v),\right. \\
\left.\sum_{k=0}^{n} \sum_{l=0}^{m} \mathbf{b}_{k l} B_{k}^{n}(u)\left(B_{l-1}^{m-1}(v)-B_{l}^{m-1}(v)\right), \sum_{p=0}^{n} \sum_{q=0}^{m} \mathbf{b}_{p q} B_{p}^{n}(u) B_{q}^{m}(v)\right) d u d v \\
=\frac{n m}{3} \sum_{i=0}^{n} \sum_{j=0}^{m} \sum_{k=0}^{n} \sum_{l=0}^{m} \sum_{p=0}^{n} \sum_{q=0}^{m}\left(\mathbf{b}_{i j}, \mathbf{b}_{k l}, \mathbf{b}_{p q}\right) \int_{0}^{1} \int_{0}^{1}\left(B_{i-1}^{n-1}(u)-B_{i}^{n-1}(u)\right) \\
B_{k}^{n}(u) B_{p}^{n}(u)\left(B_{l-1}^{m-1}(v)-B_{l}^{m-1}(v)\right) B_{j}^{m}(v) B_{q}^{m}(v) d u d v .
\end{gathered}
$$


The double integrals of the sum have a closed form but the sum contains many superfluous terms, namely $((n+1)(m+1))^{3}$. Taking into consideration the equalities

$$
\begin{aligned}
\left(\mathbf{b}_{i j}, \mathbf{b}_{k l}, \mathbf{b}_{p q}\right) & =\left(\mathbf{b}_{k l}, \mathbf{b}_{p q}, \mathbf{b}_{i j}\right)=\left(\mathbf{b}_{p q}, \mathbf{b}_{i j}, \mathbf{b}_{k l}\right)= \\
-\left(\mathbf{b}_{k l}, \mathbf{b}_{i j}, \mathbf{b}_{p q}\right) & =-\left(\mathbf{b}_{p q}, \mathbf{b}_{k l}, \mathbf{b}_{i j}\right)=-\left(\mathbf{b}_{i j}, \mathbf{b}_{p q}, \mathbf{b}_{k l}\right)
\end{aligned}
$$

and the property that a mixed product is zero if at least two of its operands are equal, we can reduce the number of terms in the sum to $\left(\begin{array}{c}(n+1)(m+1) \\ 3\end{array}\right)$.

This sum consists of the terms

$$
\begin{array}{r}
\left(\mathbf{b}_{i j}, \mathbf{b}_{k l}, \mathbf{b}_{p q}\right) \int_{0}^{1} \int_{0}^{1}\left(A_{1}(u, v)+A_{2}(u, v)+A_{3}(u, v)-\right. \\
\left.A_{4}(u, v)-A_{5}(u, v)-A_{6}(u, v)\right) d u d v
\end{array}
$$

where

$$
\begin{aligned}
& A_{1}(u, v)=\left(B_{i-1}^{n-1}(u)-B_{i}^{n-1}(u)\right) B_{j}^{m}(v)\left(B_{l-1}^{m-1}(v)-B_{l}^{m-1}(v)\right) \\
& B_{k}^{n}(u) B_{p}^{n}(u) B_{q}^{m}(v), \\
& A_{2}(u, v)=\left(B_{k-1}^{n-1}(u)-B_{k}^{n-1}(u)\right) B_{l}^{m}(v)\left(B_{q-1}^{m-1}(v)-B_{q}^{m-1}(v)\right) \\
& B_{p}^{n}(u) B_{i}^{n}(u) B_{j}^{m}(v), \\
& A_{3}(u, v)=\left(B_{p-1}^{n-1}(u)-B_{p}^{n-1}(u)\right) B_{q}^{m}(v)\left(B_{j-1}^{m-1}(v)-B_{j}^{m-1}(v)\right) \\
& B_{i}^{n}(u) B_{k}^{n}(u) B_{l}^{m}(v) \text {, } \\
& A_{4}(u, v)=\left(B_{k-1}^{n-1}(u)-B_{k}^{n-1}(u)\right) B_{l}^{m}(v)\left(B_{j-1}^{m-1}(v)-B_{j}^{m-1}(v)\right) \\
& B_{i}^{n}(u) B_{p}^{n}(u) B_{q}^{m}(v), \\
& A_{5}(u, v)=\left(B_{p-1}^{n-1}(u)-B_{p}^{n-1}(u)\right) B_{q}^{m}(v)\left(B_{l-1}^{m-1}(v)-B_{l}^{m-1}(v)\right) \\
& B_{k}^{n}(u) B_{i}^{n}(u) B_{j}^{m}(v) \text {, } \\
& A_{6}(u, v)=\left(B_{i-1}^{n-1}(u)-B_{i}^{n-1}(u)\right) B_{j}^{m}(v)\left(B_{q-1}^{m-1}(v)-B_{q}^{m-1}(v)\right) \\
& B_{p}^{n}(u) B_{k}^{n}(u) B_{l}^{m}(v) \text {. }
\end{aligned}
$$

After some simplification we gain

$$
\begin{aligned}
& A_{1}(u, v)-A_{6}(u, v)=\left(B_{i-1}^{n-1}(u)-B_{i}^{n-1}(u)\right) B_{k}^{n}(u) B_{p}^{n}(u) \\
& \left(B_{l-1}^{m-1}(v) B_{q}^{m-1}(v)-B_{q-1}^{m-1}(v) B_{l}^{m-1}(v)\right) B_{j}^{m}(v)= \\
& \left(\begin{array}{l}
n \\
k
\end{array}\right)\left(\begin{array}{l}
n \\
p
\end{array}\right)\left(\frac{\left(\begin{array}{c}
n-1 \\
i-1
\end{array}\right)}{\left(\begin{array}{c}
3 n-1 \\
i+k+p-1
\end{array}\right)} B_{i+k+p-1}^{3 n-1}(u)-\frac{\left(\begin{array}{c}
n-1 \\
i
\end{array}\right)}{\left(\begin{array}{c}
3 n-1 \\
i+k+p
\end{array}\right)} B_{i+k+p}^{3 n-1}(u)\right) \\
& \frac{\left(\begin{array}{c}
m \\
j
\end{array}\right)\left(\left(\begin{array}{c}
m-1 \\
l-1
\end{array}\right)\left(\begin{array}{c}
m-1 \\
q
\end{array}\right)-\left(\begin{array}{c}
m-1 \\
l
\end{array}\right)\left(\begin{array}{c}
m-1 \\
q-1
\end{array}\right)\right)}{\left(\begin{array}{c}
3 m-2 \\
j+l+q-1
\end{array}\right)} B_{j+l+q-1}^{3 m-2}(v) .
\end{aligned}
$$


Analogously we can obtain

$$
\begin{aligned}
& A_{2}(u, v)-A_{4}(u, v)= \\
& \left(\begin{array}{c}
n \\
i
\end{array}\right)\left(\begin{array}{l}
n \\
p
\end{array}\right)\left(\frac{\left(\begin{array}{c}
n-1 \\
k-1
\end{array}\right)}{\left(\begin{array}{c}
3 n-1 \\
i+k+p-1
\end{array}\right)} B_{i+k+p-1}^{3 n-1}(u)-\frac{\left(\begin{array}{c}
n-1 \\
k
\end{array}\right)}{\left(\begin{array}{c}
3 n-1 \\
i+k+p
\end{array}\right)} B_{i+k+p}^{3 n-1}(u)\right) \\
& \frac{\left(\begin{array}{c}
m \\
l
\end{array}\right)\left(\left(\begin{array}{c}
m-1 \\
q-1
\end{array}\right)\left(\begin{array}{c}
m-1 \\
j
\end{array}\right)-\left(\begin{array}{c}
m-1 \\
j-1
\end{array}\right)\left(\begin{array}{c}
m-1 \\
q
\end{array}\right)\right)}{\left(\begin{array}{c}
3 m-2 \\
j+l+q-1
\end{array}\right)} B_{j+l+q-1}^{3 m-2}(v),
\end{aligned}
$$

and

$$
\begin{aligned}
& A_{3}(u, v)-A_{5}(u, v)= \\
& \left(\begin{array}{c}
n \\
i
\end{array}\right)\left(\begin{array}{c}
n \\
k
\end{array}\right)\left(\frac{\left(\begin{array}{c}
n-1 \\
p-1
\end{array}\right)}{\left(\begin{array}{c}
3 n-1 \\
i+k+p-1
\end{array}\right)} B_{i+k+p-1}^{3 n-1}(u)-\frac{\left(\begin{array}{c}
n-1 \\
p
\end{array}\right)}{\left(\begin{array}{c}
3 n-1 \\
i+k+p
\end{array}\right)} B_{i+k+p}^{3 n-1}(u)\right) \\
& \frac{\left(\begin{array}{c}
m \\
q
\end{array}\right)\left(\left(\begin{array}{c}
m-1 \\
j-1
\end{array}\right)\left(\begin{array}{c}
m-1 \\
l
\end{array}\right)-\left(\begin{array}{c}
m-1 \\
j
\end{array}\right)\left(\begin{array}{c}
m-1 \\
l-1
\end{array}\right)\right)}{\left(\begin{array}{c}
3 m-2 \\
j+l+q-1
\end{array}\right)} B_{j+l+q-1}^{3 m-2}(v) .
\end{aligned}
$$

Utilizing that $\int_{0}^{1} B_{i}^{n}(u) d u=1 /(n+1),(i=0,1, \ldots, n)$ cf. [2] we gain

$$
\begin{array}{r}
\int_{0}^{1} \int_{0}^{1} A_{1}(u, v)-A_{6}(u, v) d u d v=\frac{1}{3 n(3 m-1)} \frac{1}{\left(\begin{array}{c}
3 m-2 \\
j+l+q-1
\end{array}\right)} \\
\left(\begin{array}{c}
n \\
k
\end{array}\right)\left(\begin{array}{l}
n \\
p
\end{array}\right)\left(\frac{\left(\begin{array}{c}
n-1 \\
i-1
\end{array}\right)}{\left(\begin{array}{c}
3 n-1 \\
i+k+p-1
\end{array}\right)}-\frac{\left(\begin{array}{c}
n-1 \\
i
\end{array}\right)}{\left(\begin{array}{c}
3 n-1 \\
i+k+p
\end{array}\right)}\right) \\
\left(\begin{array}{c}
m \\
j
\end{array}\right)\left(\left(\begin{array}{c}
m-1 \\
l-1
\end{array}\right)\left(\begin{array}{c}
m-1 \\
q
\end{array}\right)-\left(\begin{array}{c}
m-1 \\
l
\end{array}\right)\left(\begin{array}{c}
m-1 \\
q-1
\end{array}\right)\right) .
\end{array}
$$


Simplifying the binomials above (cf. [4]) we obtain

$$
\begin{aligned}
& \int_{0}^{1} \int_{0}^{1} A_{1}(u, v)-A_{6}(u, v) d u d v= \\
& \begin{array}{c}
(l-q)(2 i-k-p) \\
3 m-2 \\
3 n(3 m-1) m(3 n-1)\left(\begin{array}{c}
3+q-1 \\
j+l+2
\end{array}\right)\left(\begin{array}{c}
3 n-2 \\
i+k+p-1
\end{array}\right)
\end{array} \\
& \left(\begin{array}{c}
n \\
i
\end{array}\right)\left(\begin{array}{c}
n \\
k
\end{array}\right)\left(\begin{array}{c}
n \\
p
\end{array}\right)\left(\begin{array}{c}
m \\
j
\end{array}\right)\left(\begin{array}{c}
m \\
l
\end{array}\right)\left(\begin{array}{c}
m \\
q
\end{array}\right) .
\end{aligned}
$$

The integral of the differences $A_{2}(u, v)-A_{4}(u, v)$ and $A_{3}(u, v)-A_{5}(u, v)$ can analogously be expressed and we obtain the equations

$$
\begin{aligned}
& \int_{0}^{1} \int_{0}^{1} A_{2}(u, v)-A_{4}(u, v) d u d v= \\
& \begin{array}{c}
(q-j)(2 k-i-p) \\
3 m-2 \\
j n(3 m-1) m(3 n-1)\left(\begin{array}{c}
3+q-1
\end{array}\right)\left(\begin{array}{c}
3 n-2 \\
i+k+p-1
\end{array}\right)
\end{array} \\
& \left(\begin{array}{c}
n \\
i
\end{array}\right)\left(\begin{array}{c}
n \\
k
\end{array}\right)\left(\begin{array}{c}
n \\
p
\end{array}\right)\left(\begin{array}{c}
m \\
j
\end{array}\right)\left(\begin{array}{c}
m \\
l
\end{array}\right)\left(\begin{array}{c}
m \\
q
\end{array}\right)
\end{aligned}
$$

and

$$
\begin{aligned}
& \int_{0}^{1} \int_{0}^{1} A_{3}(u, v)-A_{5}(u, v) d u d v= \\
& \begin{array}{c}
(j-l)(2 p-i-k) \\
3 m-2 \\
3 n(3 m-1) m(3 n-1)\left(\begin{array}{c}
3+l+q-1 \\
j+l
\end{array}\right)\left(\begin{array}{c}
3 n-2 \\
i+k+p-1
\end{array}\right)
\end{array} \\
& \left(\begin{array}{c}
n \\
i
\end{array}\right)\left(\begin{array}{c}
n \\
k
\end{array}\right)\left(\begin{array}{c}
n \\
p
\end{array}\right)\left(\begin{array}{c}
m \\
j
\end{array}\right)\left(\begin{array}{c}
m \\
l
\end{array}\right)\left(\begin{array}{c}
m \\
q
\end{array}\right) .
\end{aligned}
$$

The sum of the integrals (2.1), (2.2) and (2.3) is

$$
\begin{aligned}
\frac{l(i-p)+q(k-i)+j(p-k)}{n(3 n-1) m(3 m-1)\left(\begin{array}{c}
3 n-2 \\
i+k+p-1
\end{array}\right)\left(\begin{array}{c}
3 m-2 \\
j+l+q-1
\end{array}\right)} \\
\left(\begin{array}{c}
n \\
i
\end{array}\right)\left(\begin{array}{l}
n \\
k
\end{array}\right)\left(\begin{array}{l}
n \\
p
\end{array}\right)\left(\begin{array}{l}
m \\
j
\end{array}\right)\left(\begin{array}{l}
m \\
l
\end{array}\right)\left(\begin{array}{l}
m \\
q
\end{array}\right) .
\end{aligned}
$$

The choice of the point triplets $\mathbf{b}_{i j}, \mathbf{b}_{k l}, \mathbf{b}_{p q}$ is still left. For this we transform the matrix with elements $\mathbf{b}_{i j},(i=0,1, \ldots, n ; j=0,1, \ldots, m)$ to a vector $\mathbf{c}_{r},(r=$ $0,1, \ldots, z-1)$. This vector has $z=(n+1)(m+1)$ components. The sum of the required triplets can be gained by the triple summation

$$
\sum_{r=0}^{z-3} \sum_{s=r+1}^{z-2} \sum_{t=s+1}^{z-1}\left(\mathbf{c}_{r}, \mathbf{c}_{s}, \mathbf{c}_{t}\right) .
$$


The subscripts of the original matrix can be calculated from $r, s, t$ by means of the equalities

$$
\begin{aligned}
j & =r \bmod (m+1), i=(r-j) /(m+1), \\
l & =s \bmod (m+1), k=(s-l) /(m+1), \\
q & =t \bmod (m+1), p=(t-q) /(m+1),
\end{aligned}
$$

which completes the proof.

Although the formula looks a bit involved, it is pleasing due to its symmetry in $n$ and $m$. Moreover, it is easy to implement in a programming language, and the program based on this formula will be efficient since the number of terms in the sum is minimized.

\section{Conclusions}

By means of Theorem 2 one can compute the volume of any solid, the boundary of which can be decomposed to Bézier surfaces. Moreover, we can compute the signed volume of the solid bounded by a B-spline patch and the cones determined by its boundary curves and the origin. In order to do this, we generate the Bézier points of the B-spline patch, cf. [1], i.e. we decompose the B-spline patch to Bézier surfaces. The sum of the signed volumes of the solids determined by these Bézier surfaces is the signed volume of the solid determined by the B-spline patch.

\section{REFERENCES}

[1] Böнm, W.: Generating the Bézier points of B-spline curves and surfaces, ComputerAided Design, 13(6), (1981), 365-366.

[2] FARIN, G.: Curves and Surfaces for Computer Aided Geometric Design, Academic Press, New York, 1988.

[3] JuhÁsz, I.: Cubic parametric curves of given tangent and curvature, Computer-Aided Design, 30(1), (1998), 1-9.

[4] Knuth, D.: The Art of Computer Programming, Addison-Wesley, Reading, 1969. 